\title{
Secrecy Performance of Eigendecomposition-Based FTN Signaling and NOFDM in Quasi-Static Fading Channel
}

\author{
Shinya Sugiura, Senior Member, IEEE
}

\begin{abstract}
In this paper, we investigate the informationtheoretic secrecy performance of recent precoded faster-thanNyquist signaling (FTN) with the aid of optimal power allocation in eigenspace. More specifically, the secrecy rate and secrecy outage probability of a fading wiretap channel, which was derived for classical Nyquist-based orthogonal signaling transmission, is extended to those of our eigendecompsition-based FTN (EFTN) signaling for a quasi-static frequency-flat Rayleigh fading channel. Our performance results demonstrate that the proposed E-FTN signaling scheme exhibits improvements in secrecy rate and secrecy outage probability over conventional Nyquist-based and FTN signaling transmissions. We also show that the same benefits as those of single-carrier E-FTN signaling are attainable by its non-orthogonal multicarrier counterpart, where subcarrier spacing is set lower than that of orthogonal frequency-division multiplexing.
\end{abstract}

Index Terms-Capacity, faster-than-Nyquist signaling, information rate, mutual information, non-orthogonal frequencydivision multiplexing, physical layer security, power allocation, secrecy rate, eigendecomposition, spectrally efficient frequencydivision multiplexing, truncation.

\section{INTRODUCTION}

$\mathbf{P}$ HYSICAL-layer security has potential advantages over classical cryptography implemented in upper layers [15]. Under the assumption that potential eavesdroppers have limited computational capabilities, efficient and secure transmissions have been established with the aid of encryption. However, perfect security from the perspective of information theory is achieved by physical-layer security, i.e., informationtheoretic security. This may function as a complement to cryptography.

In the seminal study by Wyner [6], the concept of a wiretap channel was introduced, where confidential communication between a transmitter and a receiver was considered in the presence of an eavesdropper. More specifically, in [6], under the assumption that the channel of an eavesdropper is a

Postprint accepted on 1 April 2021 for publication in IEEE Transactions on Wireless Communications (DOI: 10.1109/TWC.2021.3070891) (C) 2021 IEEE. Personal use of this material is permitted. Permission from IEEE must be obtained for all other uses, in any current or future media, including reprinting/republishing this material for advertising or promotional purposes, creating new collective works, for resale or redistribution to servers or lists, or reuse of any copyrighted component of this work in other works.

S. Sugiura is with the Institute of Industrial Science, The University of Tokyo, Tokyo 153-8505, Japan. (e-mail: sugiura@iis.u-tokyo.ac.jp). (Corresponding author: Shinya Sugiura.)

The present study was supported in part by the Japan Society for the Promotion of Science (JSPS) KAKENHI (Grant Numbers 16KK0120, 17H03259, 17K18871) and in part by JST PRESTO (Grant Number JPMJPR1933). degraded version of that of a legitimate receiver, the rate of secure communication is bounded by the difference in mutual information between a legitimate receiver (mutual information $I_{\mathrm{M}}$ ) and an eavesdropper (mutual information $I_{\mathrm{W}}$ ), which is formulated as $I_{\mathrm{S}}=\max \left(I_{\mathrm{M}}-I_{\mathrm{W}}, 0\right)$. In [7], this secrecy rate was extended to a Gaussian channel, under the same assumption that the eavesdropper has a degraded channel.

In the 2000s, information-theoretic security was rediscovered, and its achievable performance in fading channels was investigated [8-10]. In $[8,9]$, the secrecy rate of a fading channel was also defined as the difference of two mutual information measures maximized over input distributions. In [10], Bloch et al. characterized the secrecy outage probability of a wiretap fading channel and demonstrated that the average secrecy rate is positive even when the average signal-to-noise ratio (SNR) of the legitimate channel is lower than that of the eavesdropper's channel.

The main drawback of the above-mentioned secure transmission is a reduced information rate due to the presence of an eavesdropper channel. In order to combat this limitation [1116], an information-theoretic framework was presented for attaining physical-layer security in wireless multiple-input multiple-output (MIMO) links. In [11], Hero considered the security of broadcast MIMO channels for the first time, as well as the so-called constraints of a low probability of detection and a low probability of intercept, employing a different assumption than a wiretap channel. Furthermore, in [14], the secrecy rate of a broadcast MIMO wiretap channel was derived as a generalization of that of the single-antenna counterpart.

Classically, wireless communication systems have relied on orthogonal resource allocation in the time, frequency, and spatial domains in order to make it feasible to detect information symbols without suffering from inter-channel interference. However, by allowing interference-induced nonorthogonal resource allocation, more flexible transmitter design becomes possible. In faster-than-Nyquist (FTN) signaling [17-21], single-carrier symbols are transmitted with an interval smaller than that defined by the Nyquist criterion, hence enhancing the transmission rate at the cost of an increased detection complexity imposed due to inter-symbol interference (ISI). Several information-theoretic studies were then carried out for FTN signaling [22-24]. FTN signaling is capable of exploiting the excess bandwidth imposed when using a realistic band-limiting shaping filter, whereas Nyquist-based signaling is not [22]. This implies that, if both the schemes employ, for example, a root-raised cosine (RRC) shaping filter, 


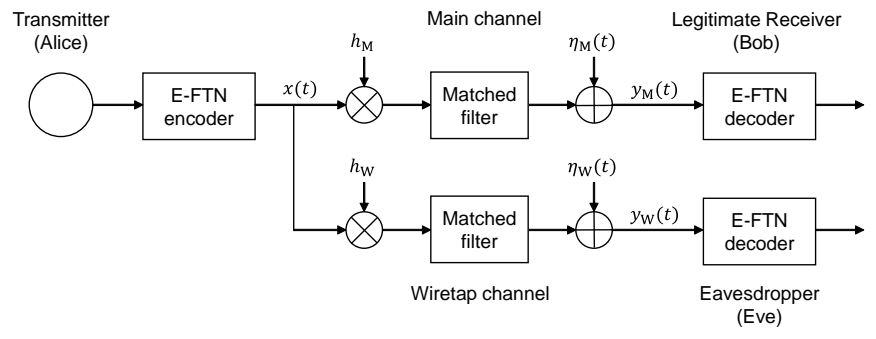

Fig. 1. System model of the proposed scheme under a fading Gaussian wiretap channel.

which has excess bandwidth, the FTN signaling may exhibit an information-theoretic gain over the Nyquist-based counterpart. Furthermore, a frequency-domain counterpart of FTN signaling was independently developed as spectrally efficient frequency-division multiplexing (SEFDM) [25-30], where subcarrier spacing in a multicarrier systems is set smaller than that of orthogonal frequency-division multiplexing (OFDM). Moreover, FTN signaling was applied to secure information transmissions [31,32]. In [31], a shaping filter employed at an FTN transmitter was hopped in time to interfere with the signal reception of the eavesdropper, where the secrecy performance was investigated under the assumption that the hopped filter coefficients are unknown at the eavesdropper. In [32], the concept of the variable symbol duration was introduced to FTN signaling for the sake of enhancing the secrecy performance, while assuming that information of the varied symbol duration is not acquired at the eavesdropper.

Most recently, in [33], Ishihara and Sugiura proposed the concept of eigendecomposition-based FTN (E-FTN) signaling, which uses precoding and optimal power allocation in eigenspace of FTN signaling. Also in [33], by extending the orthogonal-pulse assumption employed in the derivation of the classic capacity formula to the non-orthogonal case, the capacity of E-FTN signaling was formulated under the idealistic assumption that the interference matrix of FTN signaling is full rank even when the block length goes to infinity. Motivated by the E-FTN signaling scheme of [33], its frequencydomain counterpart, referred to as eigendecomposition-based non-orthogonal frequency-division multiplexing (E-NOFDM), was proposed in $[34,35]$. In E-NOFDM, spacing between subcarriers is set smaller than in OFDM, while employing eigendecomposition-aided precoding and optimal power allocation similar to [33].

The novel contributions of the present research are as follows. We investigate the security performance of the recent E-FTN signaling and E-NOFDM schemes. More specifically, we derive the secrecy rate and the secrecy outage probability for a quasi-static frequency-flat Rayleigh fading channel, as an extension of that of classical Nyquist-criterion-based orthogonal signaling. It is revealed that the secrecy rate of the E-FTN signaling increases upon increasing the packing density of time-domain symbols, and that the E-FTN signaling scheme outperforms the conventional FTN signaling and the Nyquist-based signaling benchmarks. The remainder of this paper is organized as follows. In Sections II and III, we introduce the system model of the proposed E-FTN signaling and E-NOFDM transmissions, respectively. In Section IV, the associated mutual information of the main channel and that of the wiretap channel are provided. In Section V, we derive the secrecy rate and the secrecy outage probability of the proposed scheme, and Section VI provides our performance results. Finally, we conclude this paper in Section VII.

\section{System Model OF E-FTN SignAling}

In this paper, we consider the wireless system portrayed in Fig. 1, where a transmitter (Alice) sends messages to a legitimate receiver (Bob) through a fading channel, while a single eavesdropper (Eve) intercepts the messages through another independent fading channel. We assume that both the channel of the legitimate receiver and that of the eavesdropper experience quasi-static frequency-flat independent Rayleigh fading. Each node is equipped with a single antenna element.

\section{A. Signals Transmitted from the Transmitter}

In the proposed architecture, signals transmitted from the transmitter are constituted by single-carrier E-FTN signaling [33]. Let us consider an $N$-length complex-valued information block of symbols $\mathbf{s}=\left[s_{0}, s_{1}, \cdots, s_{N-1}\right]^{T} \in \mathbb{C}^{N}$, where we have a constraint of $\mathbb{E}\left[\left|s_{i}\right|^{2}\right]=\sigma_{s}^{2}(i=0, \cdots, N-$ $1)$. The symbol block $\mathbf{s}$ is multiplied by a linear precoding matrix $\mathbf{F} \in \mathbb{C}^{N \times N}$, and hence we have the precoded symbols as follows:

$$
\begin{aligned}
\mathbf{x} & =\left[x_{0}, x_{1}, \cdots, x_{N-1}\right]^{T} \in \mathbb{C}^{N} \\
& =\mathbf{F s},
\end{aligned}
$$

where the precoding matrix $\mathbf{F}$ is designed to maintain $\mathbb{E}\left[\left|x_{i}\right|^{2}\right]=\sigma_{s}^{2}$. Then, the precoded symbols $\mathbf{x}$ are passed through a pulse-shaping filter having an impulse response $\xi(t)$ with a unit energy of $\int_{-\infty}^{\infty}|\xi(t)|^{2} d t=1$. Next, the precoded symbols are transmitted at intervals of $T=\tau T_{0}$, where $\tau$ is a packing ratio $(0<\tau \leq 1)$, and $T_{0}$ is the symbol interval defined by the Nyquist criterion. Hence, the timedomain signals of the precoded E-FTN signaling $x(t)$ are represented by

$$
x(t)=\sum_{n} x_{n} \xi\left(t-n \tau T_{0}\right) .
$$

Note that the case with $\tau=1$ corresponds to the ISIfree orthogonal Nyquist-transmission scenario. Additionally, note that except for the use of the precoding matrix, the transmitter model of E-FTN signaling is the same as that of the conventional FTN signaling. Throughout the paper, we assume the use of an RRC shaping filter with a roll-off factor $\beta$ for $\xi(t)$ unless otherwise noted.

\section{B. Signals Received at the Legitimate Receiver}

Assuming the scenario of the quasi-static frequency-flat Rayleigh fading channel and the use of a matched filter $\xi^{*}(-t)$, the signals received at the legitimate receiver, after matched filtering, can be represented by

$$
y_{\mathrm{M}}(t)=h_{\mathrm{M}} \sum_{n} x_{n} g\left(t-n \tau T_{0}\right)+\eta_{\mathrm{M}}(t),
$$




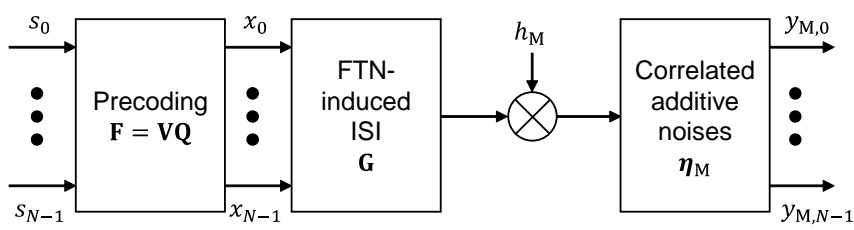

(a) General block model with power allocation

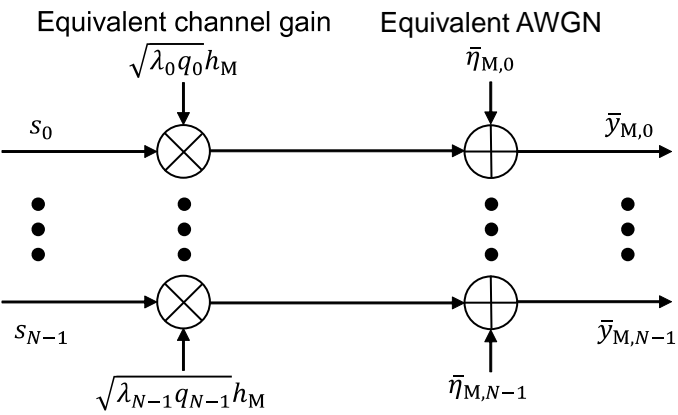

(b) Equivalent parallel channel model with power allocation

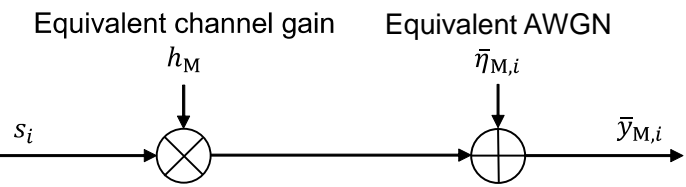

(c) Equivalent channel model with optimal power allocation

Fig. 2. Received signal models with the proposed power allocation.

where

$$
\begin{aligned}
g(t) & =\int \xi(\zeta) \xi^{*}(\zeta-t) d \zeta, \\
\eta_{\mathrm{M}}(t) & =\int n_{\mathrm{M}}(\zeta) \xi^{*}(\zeta-t) d \zeta,
\end{aligned}
$$

while $n_{\mathrm{M}}(t)$ is the associated additive white Gaussian noise (AWGN), represented by a complex-valued white Gaussian random process with a zero mean and a spectral density of $N_{0}$. Also, $h_{\mathrm{M}} \in \mathbb{C}$ is the associated channel coefficient between the transmitter and receiver, which is assumed to remain constant over each block duration and obeys the zero-mean unit-variance complex-valued Gaussian distribution. Here, the instantaneous and average SNRs at the legitimate receiver are defined respectively by

$$
\begin{aligned}
& \gamma_{\mathrm{M}}=\frac{\sigma_{s}^{2}\left|h_{\mathrm{M}}\right|^{2}}{N_{0}}, \\
& \bar{\gamma}_{\mathrm{M}}=\frac{\sigma_{s}^{2} \mathbb{E}\left[\left|h_{\mathrm{M}}\right|^{2}\right]}{N_{0}},
\end{aligned}
$$

where $\mathbb{E}[\cdot]$ is the expectation operation.

Assuming sampling of the received signals $y_{\mathrm{M}}(t)$ at intervals $\tau T_{0}$, the $i$ th received sample $y_{\mathrm{M}, i}=y_{\mathrm{M}}\left(i \tau T_{0}\right)(i=$ $0, \cdots, N-1)$ can be expressed as

$$
y_{\mathrm{M}, i}=h_{\mathrm{M}} \sum_{n} x_{n} g_{i-n}+\eta_{\mathrm{M}, i},
$$

where we have $g_{i-n}=g\left((i-n) \tau T_{0}\right)$ and $\eta_{\mathrm{M}, i}=\eta_{\mathrm{M}}\left(i \tau T_{0}\right)$. Note that $\eta_{\mathrm{M}, i}$ is a colored noise with a correlation of
$\mathbb{E}\left[\eta_{\mathrm{M}, l} \eta_{\mathrm{M}, m}^{*}\right]=N_{0} g_{l-m}$. Moreover, as shown in Fig. 2(a), the received samples of (9) have the block representation

$$
\begin{aligned}
\mathbf{y}_{\mathrm{M}} & =\left[y_{\mathrm{M}, 0}, \cdots, y_{\mathrm{M}, N-1}\right]^{T} \in \mathbb{C}^{N} \\
& =h_{\mathrm{M}} \mathbf{G x}+\boldsymbol{\eta}_{\mathrm{M}},
\end{aligned}
$$

where $\mathbf{G} \in \mathbb{R}^{N \times N}$ is the symmetric matrix, representing FTNinduced ISI, whose $i$ th-row and $j$ th-column element is given by $g_{i-j}$. Note that it is guaranteed that $\mathbf{G}$ is positive definite for a finite block length $N[24,36]$. Furthermore, we have $\boldsymbol{\eta}_{\mathrm{M}}=\left[\eta_{\mathrm{M}, 0}, \cdots, \eta_{\mathrm{M}, N-1}\right]^{T} \in \mathbb{C}^{N}$.

\section{Signals Received at the Eavesdropper}

Similar to the signals sampled at the legitimate receiver (11), those of the eavesdropper are given by

$$
\begin{aligned}
\mathbf{y}_{\mathrm{W}} & =\left[y_{\mathrm{W}, 0}, \cdots, y_{\mathrm{W}, N-1}\right]^{T} \in \mathbb{C}^{N} \\
& =h_{\mathrm{W}} \mathbf{G x}+\boldsymbol{\eta}_{\mathrm{W}},
\end{aligned}
$$

where $y_{\mathrm{W}, i} \in \mathbb{C}$ is the $i$ th sampled signal, and $h_{\mathrm{W}} \in \mathbb{C}$ is the channel coefficient of the frequency-flat independent Rayleigh fading channel between the transmitter and the eavesdropper, which obeys the zero-mean unit-variance complex-valued Gaussian distribution. The instantaneous and average SNRs at the eavesdropper are given by

$$
\begin{aligned}
& \gamma_{\mathrm{W}}=\frac{\sigma_{s}^{2}\left|h_{\mathrm{W}}\right|^{2}}{N_{0}} \\
& \bar{\gamma}_{\mathrm{W}}=\frac{\sigma_{s}^{2} \mathbb{E}\left[\left|h_{\mathrm{W}}\right|^{2}\right]}{N_{0}},
\end{aligned}
$$

respectively. Moreover, $\boldsymbol{\eta}_{\mathrm{W}}=\left[\eta_{\mathrm{W}, 0}, \cdots, \eta_{\mathrm{W}, N-1}\right]^{T} \in \mathbb{C}^{N}$ are the associated additive noises, which have the same statistical characteristics as $\boldsymbol{\eta}_{\mathrm{M}}$. It is assumed that the eavesdropper is time-synchronized with the transmitter, while knowing perfect channel state information $h_{\mathrm{W}}$, the block length $N$, the shaping filter $\xi(t)$, and the packing ratio $\tau$, similar to the legitimate receiver.

\section{SySTEM MOdEL OF E-NOFDM}

While in Section II, we presented the wiretap system model of E-FTN signaling, in this section, we provide its frequencydomain counterpart, based on [35]. More specifically, ENOFDM is characterized by the packing of subcarriers. As shown below, the system models of E-FTN signaling and ENOFDM share the similar structure, and hence both schemes have the same secrecy performance gain over conventional OFDM transmission.

\section{A. Signals Transmitted from the Legitimate Transmitter}

Similar to the E-FTN signaling case, let us consider information symbols $\mathbf{s}$ and their precoded symbols $\mathbf{x}=\mathbf{F s}$. In this case, the precoded symbols $\mathrm{x}$ are multiplied by nonorthogonal subcarriers in a E-NOFDM frame. The symbols are

\footnotetext{
${ }^{1}$ Since the main focus of this paper is the derivation of the theoretical secrecy bound of E-FTN signaling, the detection process of the received signals is not detailed here. Readers interested in E-FTN detection should refer to [33].
} 
placed at closer spacing than in the OFDM counterpart. More specifically, given $T^{(f)}$ as the frame duration, the subcarrier spacing of E-NOFDM is expressed as $\Delta f=\tau^{(f)} / T^{(f)}$, where $\tau^{(f)}\left(0<\tau^{(f)} \leq 1\right)$ is a subcarrier's packing ratio.

The modulated signals are represented by

$$
x^{(f)}(t)=\sum_{n=0}^{N^{(f)}-1} x_{n} \xi^{(f)}(t) e^{j 2 \pi n \Delta f t},
$$

where $\xi^{(f)}(t) \in \mathbb{R}$ is the impulse response of a shaping filter. In the proposed scheme, similar to a filter bank multicarrier (FBMC) [37], each subcarrier, rather than the whole bandwidth, is bandlimited by $\xi^{(f)}(t)$. Note that a rectangular pulse is used for $\xi^{(f)}(t)$ in the conventional OFDM. For the sake of simplicity, we consider the scenario of a single-frame transmission. Note that the special case of $\mathbf{F}=\mathbf{I}$ corresponds to the unprecoded E-NOFDM scheme, where $\mathbf{I}$ is the identity matrix.

When considering a specific available bandwidth, the relationship between the number of subcarriers of E-NOFDM and that of OFDM $N_{\text {OFDM }}$ is given by

$$
N^{(f)}=\left\lfloor\frac{N_{\mathrm{OFDM}}^{(f)}-1}{\tau^{(f)}}\right\rfloor+1 .
$$

If the available bandwidth is sufficiently wide, i.e., $N_{\mathrm{OFDM}}^{(f)} \gg$ $1, N^{(f)}$ is approximated as

$$
N^{(f)} \simeq\left\lfloor\frac{N_{\mathrm{OFDM}}^{(f)}}{\tau^{(f)}}\right\rfloor .
$$

\section{B. Signals Received at the Legitimate Receiver}

Under the assumption of a frequency-flat Rayleigh fading channel, the received signals are represented by

$$
y_{\mathrm{M}}^{(f)}(t)=h_{\mathrm{M}} x^{(f)}(t)+n_{\mathrm{M}}(t),
$$

where $n_{\mathrm{M}}(t)$ is a complex-valued white Gaussian random process with a zero mean and a spectral density of $N_{0}$. The received signals are first passed through a matched filter $\xi^{(f) *}(-t)$, and then the filtered outputs are projected onto the E-NOFDM subcarriers. More specifically, the received sample corresponding to the $i$ th subcarrier is given by

$$
y_{\mathrm{M}, i}^{(f)}=\int_{-\infty}^{\infty} y_{\mathrm{M}}^{(f)}(t) \xi^{(f) *}(-t) e^{-j 2 \pi i \Delta f t} d t \in \mathbb{C},
$$

which also has the block representation

$$
\begin{aligned}
\mathbf{y}_{\mathrm{M}}^{(f)} & =\left[y_{\mathrm{M}, 0}^{(f)}, \cdots, y_{\mathrm{M}, N-1}^{(f)}\right]^{T} \in \mathbb{C}^{N} \\
& =h_{\mathrm{M}} \mathbf{G}^{(f)} \mathbf{x}+\boldsymbol{\eta}_{\mathrm{M}}^{(f)}
\end{aligned}
$$

where the matrix $\mathbf{G}^{(f)} \in \mathbb{C}^{N \times N}$ represents the effects of inter-carrier interference (ICI), and its $a$ th-row and $b$ th-column element is [38]

$$
g_{a, b}^{(f)}=\int_{-\infty}^{\infty} \xi^{(f)}(t) \xi^{(f) *}(-t) e^{j 2 \pi(b-a) \Delta f t} d t .
$$

Furthermore, $\boldsymbol{\eta}_{\mathrm{M}}^{(f)}=\left[\eta_{\mathrm{M}, 0}^{(f)}, \cdots, \eta_{\mathrm{M}, N-1}^{(f)}\right]^{T}$ are the correlated noises, each formulated by

$$
\eta_{\mathrm{M}, i}^{(f)}=\int_{-\infty}^{\infty} n_{\mathrm{M}}(t) g^{*}(-t) e^{-j 2 \pi i \Delta f t} d t .
$$

Here, the correlation of the noises $\eta_{\mathrm{M}, i}^{(f)}$ is given by $\mathbb{E}\left[\eta_{\mathrm{M}, l}^{(f)} \eta_{\mathrm{M}, m}^{(f)}\right]=N_{0} g_{l, m}^{(f)}$. Note that the case with $\tau^{(f)}=1$ as well as a rectangular pulse corresponds to the classical OFDM, where the ICI matrix $\mathbf{G}^{(f)}$ becomes the identity matrix $\mathbf{I}$, owing to the orthogonality of subcarriers.

\section{Signals Received at the Eavesdropper}

In a similar manner to the received signal model (22) of the legitimate receiver, that of the eavesdropper is represented by

$$
\begin{aligned}
\mathbf{y}_{\mathrm{W}}^{(f)} & =\left[y_{\mathrm{W}, 0}^{(f)}, \cdots, y_{\mathrm{W}, N-1}^{(f)}\right]^{T} \in \mathbb{C}^{N} \\
& =h_{\mathrm{W}} \mathbf{G}^{(f)} \mathbf{x}+\boldsymbol{\eta}_{\mathrm{W}}^{(f)},
\end{aligned}
$$

where the associated noise components $\boldsymbol{\eta}_{\mathrm{W}}^{(f)}=$ $\left[\eta_{\mathrm{W}, 0}^{(f)}, \cdots, \eta_{\mathrm{W}, N-1}^{(f)}\right]^{T}$ are random variables having the same distribution and variance as $\boldsymbol{\eta}_{\mathrm{M}}^{(f)}$.

\section{Mutual Information of E-FTN Signaling}

In this section, we formulate the mutual information of the main and wiretap channels in the proposed E-FTN signaling and E-NOFDM schemes, which were introduced in Section II and Section III, respectively. More specifically, according to the mutual information of E-FTN signaling, derived for the AWGN scenario [33], we introduce that of the quasi-static frequency-flat fading channel. In the remainder of this paper, we only consider the scenario of E-FTN signaling transmission. However, the system models of the E-FTN signaling and E-NOFDM schemes are equivalent, as seen in (22) and (26). Hence, the E-NOFDM scheme is readily applicable simply by omitting the superscript $(f)$.

\section{A. Mutual Information of the Main Channel}

In order to simplify the mutual information derivation of E-FTN signaling, the ISI-contaminated samples at the legitimate receiver (11) are decomposed into independent parallel substreams. More specifically, since, as above-mentioned, G is positive definite for a finite block length $N, \mathbf{G}$ is factorized as

$$
\mathbf{G}=\mathbf{V} \boldsymbol{\Lambda} \mathbf{V}^{T}
$$

with the aid of eigendecomposition, where $\mathbf{V}$ are $N \times N$ orthogonal matrices and $\boldsymbol{\Lambda}=\operatorname{diag}\left\{\lambda_{0}, \cdots, \lambda_{N-1}\right\}$ is a real-valued diagonal matrix composed of descending-order eigenvalues $\lambda_{0} \geq \lambda_{1} \geq \cdots \geq \lambda_{N-1}$. More specifically, the minimum eigenvalue is positive, i.e., $\lambda_{N-1}>0$, for a finite block length $N$. Also, we have the relationship of

$$
\begin{aligned}
\sum_{i=0}^{N-1} \lambda_{i} & =\operatorname{trace}\{\mathbf{G}\} \\
& =N
\end{aligned}
$$


since each diagonal element of $\mathbf{G}$ is unity.

Furthermore, let us set the precoding matrix to $\mathbf{F}=\mathbf{V Q}$, where $\mathbf{Q}=\operatorname{diag}\left\{\sqrt{q_{0}}, \cdots, \sqrt{q_{N-1}}\right\} \in \mathbb{R}^{N}$ is a real-valued diagonal power allocation matrix. Note that the calculations of the precoding matrix $\mathbf{F}$ do not require knowledge of the channel coefficient $h_{\mathrm{M}}$ or the noise variance $N_{0}$ at the transmitter. Hence, the calculations are carried out offline in advance of transmissions. Then, (11) can be rewritten as follows:

$$
\begin{aligned}
\mathbf{y}_{\mathrm{M}} & =h_{\mathrm{M}}\left(\mathbf{V} \boldsymbol{\Lambda} \mathbf{V}^{T}\right)(\mathbf{V Q}) \mathbf{s}+\boldsymbol{\eta}_{\mathrm{M}} \\
& =h_{\mathrm{M}} \mathbf{V} \boldsymbol{\Lambda} \mathbf{Q} \mathbf{s}+\boldsymbol{\eta}_{\mathrm{M}} .
\end{aligned}
$$

At the legitimate receiver, by multiplying the weight matrix $\Lambda^{-\frac{1}{2}} \mathbf{V}^{T}$ by the received samples $\mathbf{y}_{\mathrm{M}}$, the following representation is obtained:

$$
\begin{aligned}
\overline{\mathbf{y}}_{\mathrm{M}} & =\left[\bar{y}_{\mathrm{M}, 0}, \cdots, \bar{y}_{\mathrm{M}, N-1}\right]^{T} \in \mathbb{C}^{N} \\
& =\boldsymbol{\Lambda}^{-\frac{1}{2}} \mathbf{V}^{T} \mathbf{y}_{\mathrm{M}} \\
& =h_{\mathrm{M}} \boldsymbol{\Lambda}^{\frac{1}{2}} \mathbf{Q} \mathbf{s}+\overline{\boldsymbol{\eta}}_{\mathrm{M}},
\end{aligned}
$$

where

$$
\begin{aligned}
\overline{\boldsymbol{\eta}}_{\mathrm{M}} & =\left[\bar{\eta}_{\mathrm{M}, i}, \cdots, \bar{\eta}_{\mathrm{M}, i}\right]^{T} \\
& =\boldsymbol{\Lambda}^{-\frac{1}{2}} \mathbf{V}^{T} \boldsymbol{\eta}_{\mathrm{M}},
\end{aligned}
$$

which are the uncorrelated noises with a variance of $N_{0} \mathbf{I}$ [33]. Since $\boldsymbol{\Lambda}^{\frac{1}{2}} \mathbf{Q}$ is diagonal, $\overline{\mathbf{y}}_{\mathrm{M}}$ correspond to $N$ ISI-free independent parallel substreams of

$$
\bar{y}_{\mathrm{M}, i}=\sqrt{\lambda_{i} q_{i}} h_{\mathrm{M}} s_{i}+\bar{\eta}_{\mathrm{M}, i},
$$

where the equivalent channel gain in (36) may be regarded as $\sqrt{\lambda_{i} q_{i}} h_{\mathrm{M}}$ (Fig. 2(b)).

Since the received samples of (36) do not contain the effects of ISI and the noises are uncorrelated, the upper bound of mutual information per block is given by

$$
I\left(\mathbf{s} ; \overline{\mathbf{y}}_{\mathrm{M}}\right) \leq \sum_{i=0}^{N-1} \log _{2}\left(1+\frac{\lambda_{i} q_{i} \sigma_{s}^{2}\left|h_{\mathrm{M}}\right|^{2}}{N_{0}}\right) .
$$

Furthermore, based on the Lagrange multiplier method [33], the optimal power allocation factors $q_{i}(i=0, \cdots, N-1)$ are derived, such that the mutual information of (37) is maximized. More specifically, let us consider the total transmit energy per block is calculated by

$$
\begin{aligned}
E_{\mathrm{b}} & =\mathbb{E}\left[\int_{-\infty}^{\infty}|x(t)|^{2} d t\right] \\
& =\mathbb{E}\left[\sum_{l} \sum_{m} x_{l} x_{m}^{*} \xi(t-l T) \xi(t-m T) d t\right] \\
& =\mathbb{E}\left[\sum_{l} \sum_{m} x_{l} x_{m}^{*} g((l-m) T)\right] \\
& =\mathbb{E}\left[\mathbf{x}^{H} \mathbf{G} \mathbf{x}\right] \\
& =\mathbb{E}\left[\mathbf{s}^{H} \mathbf{Q} \mathbf{V}^{T} \mathbf{G V Q} \mathbf{Q s}\right] \\
& =\mathbb{E}\left[\mathbf{s}^{H} \mathbf{Q} \mathbf{\Lambda} \mathbf{Q s}\right] \\
& =\sum_{i=0}^{N-1} \lambda_{i} q_{i} \mathbb{E}\left[\left|s_{i}\right|^{2}\right] \\
& =\sigma_{s}^{2} \sum_{i=0}^{N-1} \lambda_{i} q_{i} .
\end{aligned}
$$

For the power-unallocated scenario of $q_{0}=\cdots=q_{N-1}=1$, (45) becomes

$$
\begin{aligned}
E_{\mathrm{b}}^{\prime} & =\sigma_{s}^{2} \sum_{i=0}^{N-1} \lambda_{i} \\
& =\sigma_{s}^{2} N .
\end{aligned}
$$

Since the total power per block has to remain unchanged, regardless of the presence or absence of power allocation, we arrive at the power constraint of

$$
\begin{gathered}
E_{\mathrm{b}}=E_{\mathrm{b}}^{\prime} \\
\Leftrightarrow \sum_{i=0}^{N-1} \lambda_{i} q_{i}=N .
\end{gathered}
$$

Then, from (37) and (48), Lagrange function is defined by

$$
J=\sum_{i=0}^{N-1} \log _{2}\left(1+\frac{\lambda_{i} q_{i} \sigma_{s}^{2}\left|h_{\mathrm{M}}\right|^{2}}{N_{0}}\right)-\alpha\left(\sum_{i=0}^{N-1} \lambda_{i} q_{i}-N\right)
$$

where $\alpha$ is the Lagrange multiplier. By maximizing $J$ with respect to $q_{i}$, the optimal power allocation factors can be derived as

$$
q_{i}=\frac{1}{\lambda_{i}}(i=0, \cdots, N-1) .
$$

Note that while optimal power allocation in the proposed scheme is derived similar to that of the singular-value decomposition (SVD)-based MIMO [39], the resultant coefficients of (50) are totally different from those of the SVD-MIMO counterpart. This is because the energy (power) constraint (48) considered in the proposed scheme is different from that of the SVD-MIMO scheme.

With the aid of optimal power allocation (50), the received signal model (36) is further modified to the following (Fig. 2(c)):

$$
\bar{y}_{\mathrm{M}, i}=h_{\mathrm{M}} s_{i}+\bar{\eta}_{\mathrm{M}, i} .
$$

Also, from (37) and (50), the associated mutual information, 
which is maximized by optimal power allocation, can be represented by

$$
I_{\mathrm{M}}=N \log _{2}\left(1+\frac{\sigma_{s}^{2}\left|h_{\mathrm{M}}\right|^{2}}{N_{0}}\right) \quad[\text { bits/block }] .
$$

Finally, by normalizing (52) by the block length $N$ and the symbol interval $\tau T_{0}$, the instantaneous mutual information of the optimal power allocation scheme is rewritten by

$$
\begin{aligned}
I_{\mathrm{M}}^{\prime} & =\frac{1}{\tau T_{0} N} I_{\mathrm{M}} \\
& =\frac{1}{\tau T_{0}} \log _{2}\left(1+\frac{\sigma_{s}^{2}\left|h_{\mathrm{M}}\right|^{2}}{N_{0}}\right) \\
& =\frac{B}{\tau} \log _{2}\left(1+\gamma_{\mathrm{M}}\right)[\mathrm{bps}],
\end{aligned}
$$

where we have $B=1 / T_{0}$, noting the channel bandwidth is $(1+\beta) B$ due to the use of an RRC shaping filter with the roll-off factor of $\beta$. Note that the derived mutual information (55) does not include eigenvalues, owing to the cancellation by the optimal power allocation factors.

By averaging the instantaneous mutual information over the distribution of $\gamma_{M}$, i.e., $p\left(\gamma_{M}\right)$, the average information rate is given by

$$
\begin{aligned}
\bar{I}_{\mathrm{M}} & =\int_{0}^{\infty} I_{\mathrm{M}}^{\prime} p\left(\gamma_{\mathrm{M}}\right) d \gamma_{\mathrm{M}} \\
& =\int_{0}^{\infty} \frac{B}{\tau} \log _{2}\left(1+\gamma_{\mathrm{M}}\right) p\left(\gamma_{\mathrm{M}}\right) d \gamma_{\mathrm{M}} \\
& \leq \frac{B}{\tau} \log _{2}\left(1+\bar{\gamma}_{\mathrm{M}}\right),
\end{aligned}
$$

where Jensen's inequality [39] is used from (57) to (58). ${ }^{2}$

\section{B. Mutual Information of the Wiretap Channel}

Since the proposed E-FTN signaling scheme is based on an open-loop transmission without any feedback associated with the channel coefficient from the legitimate receiver, the received signal models of the legitimate receiver and the eavesdropper are essentially identical. Hence, similar to the above-derived mutual information of the main channel, the instantaneous mutual information and its average one of the wiretap channel are given respectively by

$$
\begin{aligned}
I_{\mathrm{W}}^{\prime} & =\frac{B}{\tau} \log _{2}\left(1+\gamma_{\mathrm{W}}\right), \\
\bar{I}_{\mathrm{W}} & =\frac{B}{\tau} \log _{2}\left(1+\bar{\gamma}_{\mathrm{W}}\right) .
\end{aligned}
$$

\section{SECRECy PERformance}

In this section, we formulate the secrecy rate and the secrecy outage probability for the proposed E-FTN signaling scheme in the fading wiretap channel while investigating the secrecy performance. As mentioned above, the secrecy performance metrics in this section is readily applicable to E-NOFDM.

\footnotetext{
${ }^{2}$ In [33], the limit of $N \rightarrow \infty$ was considered to derive the capacity, under the ideal assumption that $\mathbf{G}$ remains positive-definite regardless of $\tau$. This allows us to eliminate the effects of inter-block interference (IBI). However the validity of this assumption has not been proved in a theoretical manner, and hence in this paper only the scenario of $\tau \geq 1 /(1+\beta)$ is considered to maintain the positive definiteness of $\mathbf{G}$.
}

\section{A. Secrecy Rate}

Since, as already described, the channel coefficients in the main and wiretap channels $h_{\mathrm{M}}$ and $h_{\mathrm{W}}$ are zero-mean unitvariance complex-valued Gaussian variables, the associated probability density functions are given by

$$
\begin{aligned}
& p\left(\gamma_{\mathrm{M}}\right)=\frac{1}{\bar{\gamma}_{\mathrm{M}}} \exp \left(-\frac{\gamma_{\mathrm{M}}}{\bar{\gamma}_{\mathrm{M}}}\right), \\
& p\left(\gamma_{\mathrm{W}}\right)=\frac{1}{\bar{\gamma}_{\mathrm{W}}} \exp \left(-\frac{\gamma_{\mathrm{W}}}{\bar{\gamma}_{\mathrm{W}}}\right),
\end{aligned}
$$

respectively.

The secrecy rate is given by the difference between mutual information of the main channel and that of the wiretap channel [10]. Hence, from (55) and (59), the instantaneous secrecy rate for one realization of the quasi-static fading scenario is formulated by

$$
\begin{aligned}
I_{\mathrm{S}}^{\prime} & = \begin{cases}I_{\mathrm{M}}^{\prime}-I_{\mathrm{W}}^{\prime} & \left(I_{\mathrm{M}}^{\prime}>I_{\mathrm{W}}^{\prime}\right) \\
0 & \left(I_{\mathrm{M}}^{\prime} \leq I_{\mathrm{W}}^{\prime}\right)\end{cases} \\
& =\left\{\begin{array}{ll}
\frac{B}{\tau}\left[\log _{2}\left(1+\gamma_{\mathrm{M}}\right)-\log _{2}\left(1+\gamma_{\mathrm{W}}\right)\right] & \left(\gamma_{\mathrm{M}}>\gamma_{\mathrm{W}}\right) \\
0 & \left(\gamma_{\mathrm{M}} \leq \gamma_{\mathrm{W}}\right)
\end{array} .\right.
\end{aligned}
$$

The associated average secrecy rate is obtained by averaging over the distributions of $\gamma_{\mathrm{M}}$ and $\gamma_{\mathrm{W}}$ as follows:

$$
\bar{I}_{\mathrm{S}}=\int_{0}^{\infty} \int_{0}^{\infty} I_{\mathrm{S}}^{\prime} p\left(\gamma_{\mathrm{M}}\right) p\left(\gamma_{\mathrm{W}}\right) d \gamma_{\mathrm{M}} d \gamma_{\mathrm{W}} .
$$

\section{B. Secrecy Outage Probability}

Let us next consider the probability that the normalized instantaneous secrecy rate $I_{\mathrm{S}}^{\prime} /(B(1+\beta))$ is higher than a specific target rate $R(>0)$, which is formulated as follows:

$$
\begin{aligned}
\operatorname{Pr}\left[\frac{I_{\mathrm{S}}^{\prime}}{B(1+\beta)}>R\right] & =\operatorname{Pr}\left[\frac{1}{\tau(1+\beta)} \log _{2} \frac{1+\gamma_{\mathrm{M}}}{1+\gamma_{\mathrm{W}}}>R\right] \\
& =\operatorname{Pr}\left[\gamma_{\mathrm{M}}>2^{\tau R(1+\beta)}\left(1+\gamma_{\mathrm{W}}\right)-1\right] \quad(67) \\
& =\int_{0}^{\infty} p\left(\gamma_{\mathrm{W}}\right) \\
& \times\left(\int_{2^{\tau R(1+\beta)}\left(1+\gamma_{\mathrm{W})-1}\right.}^{\infty} p\left(\gamma_{\mathrm{M}}\right) d \gamma_{\mathrm{M}}\right) d \gamma_{\mathrm{W}},
\end{aligned}
$$

where $\operatorname{Pr}[\cdot]$ denotes the probability. Using (61) and (62), (68) can be rewritten as follows:

$$
\begin{aligned}
\operatorname{Pr}\left[\frac{I_{\mathrm{S}}^{\prime}}{B(1+\beta)}>R\right]= & \frac{\bar{\gamma}_{\mathrm{M}}}{\bar{\gamma}_{\mathrm{M}}+\bar{\gamma}_{\mathrm{W}} 2^{\tau R(1+\beta)}} \\
& \times \exp \left(-\frac{2^{\tau R(1+\beta)}-1}{\bar{\gamma}_{\mathrm{M}}}\right) .
\end{aligned}
$$


Then, the secrecy outage probability of the proposed E-FTN signaling scheme can be formulated from (69) as

$$
\begin{aligned}
P_{\text {out }}(R) & =\operatorname{Pr}\left[\frac{I_{\mathrm{S}}^{\prime}}{B(1+\beta)} \leq R\right] \\
& =1-\frac{\bar{\gamma}_{\mathrm{M}}}{\bar{\gamma}_{\mathrm{M}}+\bar{\gamma}_{\mathrm{W}} 2^{\tau R(1+\beta)}} \exp \left(-\frac{2^{\tau R(1+\beta)}-1}{\bar{\gamma}_{\mathrm{M}}}\right),
\end{aligned}
$$

which monotonically decreases upon decreasing the target rate $R$, the packing ratio $\tau$, or the roll-off factor $\beta$. For the limit of $R \rightarrow 0$, the secrecy outage probability simplifies to

$$
\lim _{R \rightarrow 0} P_{\text {out }}(R)=1-\frac{\bar{\gamma}_{\mathrm{M}}}{\bar{\gamma}_{\mathrm{M}}+\bar{\gamma}_{\mathrm{W}}},
$$

which does not depends on $\tau$ and moreover agrees with that of the conventional Nyquist-based scenario. This implies that for a significantly low target rate $(R \ll 1)$, the performance gain of the proposed E-FTN signaling scheme in terms of the secrecy outage (relative to conventional Nyquist-based sinc-pulse transmission) vanishes. By contrast, for the high target-rate limit of $\tau R \rightarrow \infty$, i.e., $R \gg 1 / \tau$, the secrecy outage probability approaches one, similar to the Nyquistbased scenario [10].

Additionally, let us consider the asymptotic behavior of the outage probability for the scenarios of significantly high SNRs of the main channel and the wiretap channel. For $\bar{\gamma}_{\mathrm{M}} \gg \bar{\gamma}_{\mathrm{W}}$, the analytical outage probability (71) is changed to

$$
\begin{aligned}
P_{\text {out }}(R) & \approx 1-\exp \left(-\frac{2^{\tau R(1+\beta)}-1}{\bar{\gamma}_{\mathrm{M}}}\right) \\
& =1-\sum_{n=0}^{\infty} \frac{\left(-\frac{2^{\tau R(1+\beta)}-1}{\bar{\gamma}_{\mathrm{M}}}\right)^{n}}{n !} .
\end{aligned}
$$

Furthermore, in a significantly high SNR of the main channel, (74) is approximated by

$$
P_{\text {out }}(R) \approx \frac{2^{\tau R(1+\beta)}-1}{\bar{\gamma}_{\mathrm{M}}},
$$

which indicates that the outage probability decays as $1 / \bar{\gamma}_{\mathrm{M}}$.

\section{Performance Results}

In this section, we provide our secrecy performance results of E-FTN signaling. The Nyquist pulse transmission with the RRC shaping filter and that with the idealistic rectangular shaping filter (sinc pulse) were considered as the benchmark scheme and the scheme showing performance bound, respectively. We assumed that the packing ratio of the proposed EFTN scheme ranged $\tau \geq 1 /(1+\beta)$. This constraint allows us to accurately calculate all the eigenvalues. Otherwise, i.e., in the packing ratio range of $\tau<1 /(1+\beta)$, there may be significantly low eigenvalues, which imposes calculation precision higher than that attainable by the standard doubleprecision environment [40]. Moreover, such a low $\tau$ scenario tends to induce unavoidable IBI, which is also an open issue. Additionally, it was shown in [33] that the E-FTN signaling scheme with optimal power allocation does not suffer from the effects of spectral broadening at least in the range of $1 /(1+\beta) \leq \tau \leq 1$.

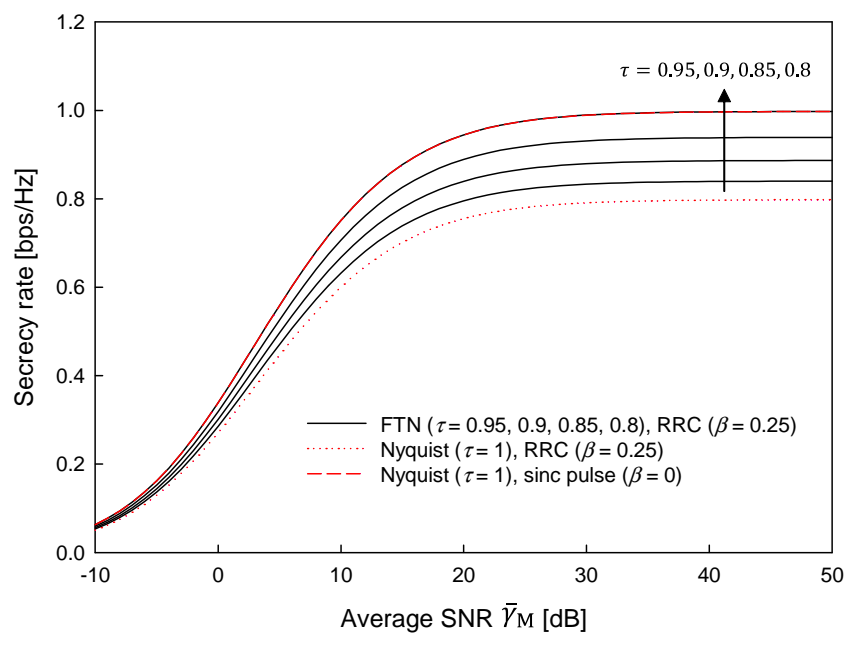

(a) $\bar{\gamma}_{\mathrm{M}}=\bar{\gamma}_{\mathrm{W}}$

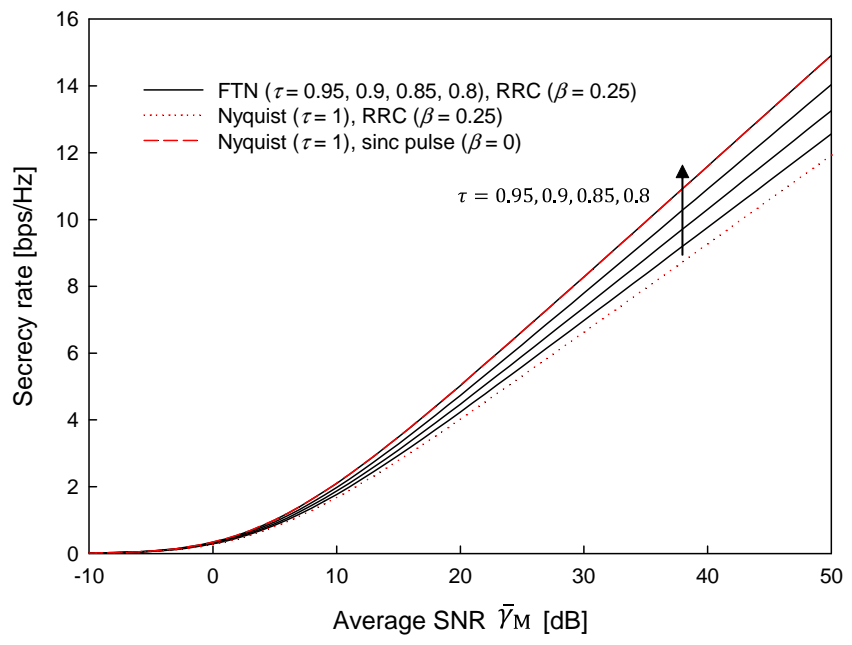

(b) $\bar{\gamma}_{\mathrm{W}}=0 \mathrm{~dB}$

Fig. 3. Numerical average secrecy rate $\mathbb{E}\left[I_{\mathrm{S}}^{\prime}\right] /(B(1+\beta)) \mathrm{bps} / \mathrm{Hz}$ of the proposed E-FTN signaling. Packing ratios from $\tau=0.95$ to 0.8 are shown. The vertical arrow indicates decreasing $\tau$. (a) $\bar{\gamma}_{\mathrm{M}}=\bar{\gamma}_{\mathrm{W}}$, (b) $\bar{\gamma}_{\mathrm{W}}=0 \mathrm{~dB}$.

\section{A. Secrecy Rate}

Fig. 3 shows the numerical average secrecy rate $\mathbb{E}\left[I_{\mathrm{S}}^{\prime}\right] /(B(1+\beta))$ bps/Hz of the proposed E-FTN signaling scheme, where Monte Carlo simulations were carried out for averaging $I_{\mathrm{S}}^{\prime}$ of (64). More than 1000000 channel realizations were generated for the calculations of each curve. In Fig. 3(a), the average SNRs of the main and wiretap channels were set identically, i.e., $\bar{\gamma}_{\mathrm{M}}=\bar{\gamma}_{\mathrm{W}}$, while in Fig. 3(b), the average SNR of the wiretap channel was maintained to be $\bar{\gamma}_{\mathrm{W}}=0$ $\mathrm{dB}$. The packing ratio of E-FTN signaling was varied from $\tau=0.95$ to 0.8 with the step of 0.05 , and the roll-off factor of the RRC shaping filter was set to $\beta=0.25$. We also calculated the associated secrecy rate curves of the conventional Nyquist transmission schemes $(\tau=1)$, which employed the RRC shaping filter and the idealistic rectangular shaping filter (sinc pulse). Observe in Fig. 3 that as the packing 


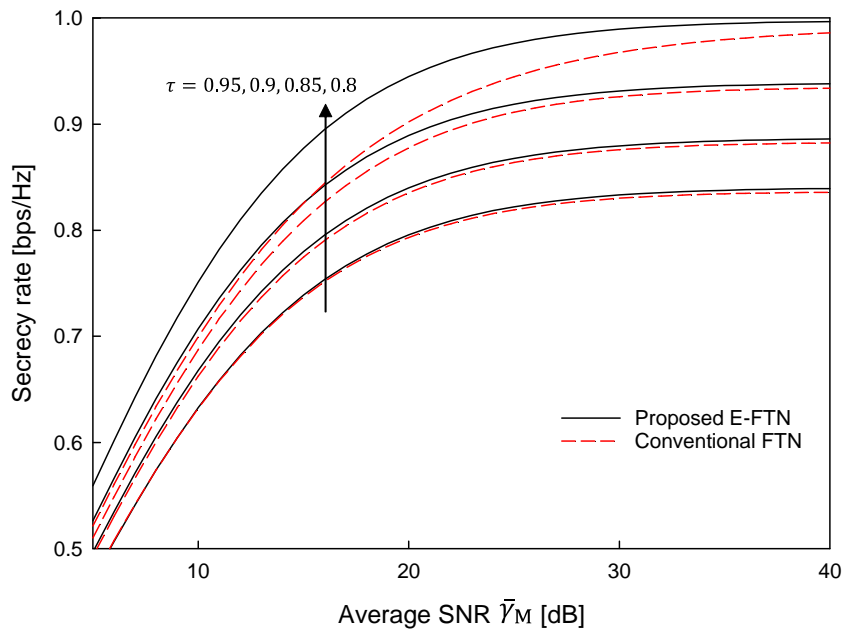

(a) $\bar{\gamma}_{\mathrm{M}}=\bar{\gamma}_{\mathrm{W}}$

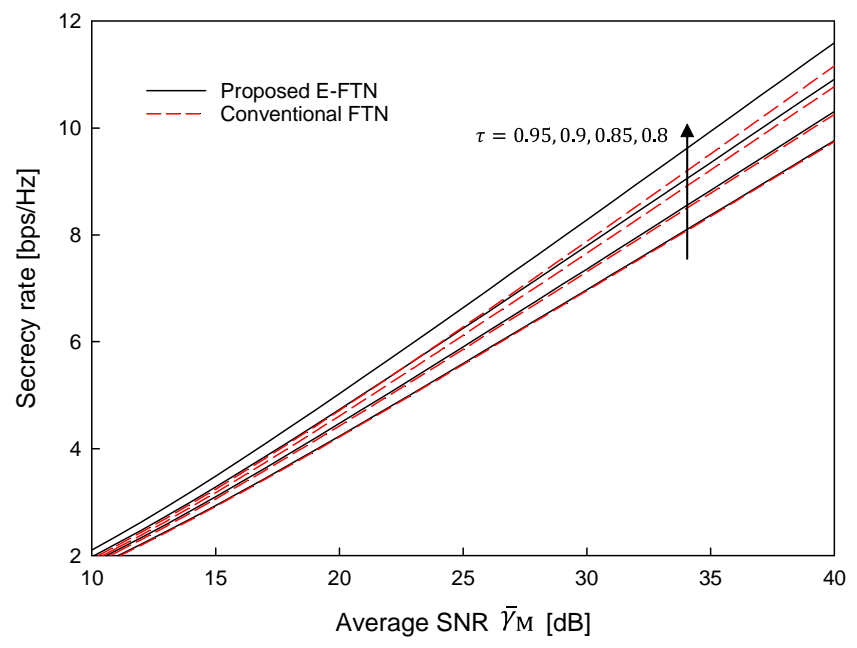

(b) $\bar{\gamma}_{\mathrm{W}}=0 \mathrm{~dB}$

Fig. 4. Numerical average secrecy rate $\mathbb{E}\left[I_{\mathrm{S}}^{\prime}\right] /(B(1+\beta)) \mathrm{bps} / \mathrm{Hz}$ of the proposed E-FTN and conventional FTN signaling schemes. Packing ratios from $\tau=0.95$ to 0.8 are shown. The vertical arrow indicates decreasing $\tau$. (a) $\bar{\gamma}_{\mathrm{M}}=\bar{\gamma}_{\mathrm{W}}$, (b) $\bar{\gamma}_{\mathrm{W}}=0 \mathrm{~dB}$.

ratio $\tau$ decreases, the average secrecy rate monotonically increased, and coincides with its idealistic bound of Nyqust sinc pulse transmission. Note that the average secrecy rate of the proposed scheme with $\tau=1$ corresponds to that of conventional Nyquist-based signaling [10].

Next, in Fig. 4, we compared the numerical average secrecy rate $\mathbb{E}\left[I_{\mathrm{S}}^{\prime}\right] /(B(1+\beta))$ bps $/ \mathrm{Hz}$ between the proposed E-FTN signaling scheme and the conventional FTN signaling benchmark without power allocation. Here, similar to (64), the instantaneous secrecy rate of the benchmark was formulated

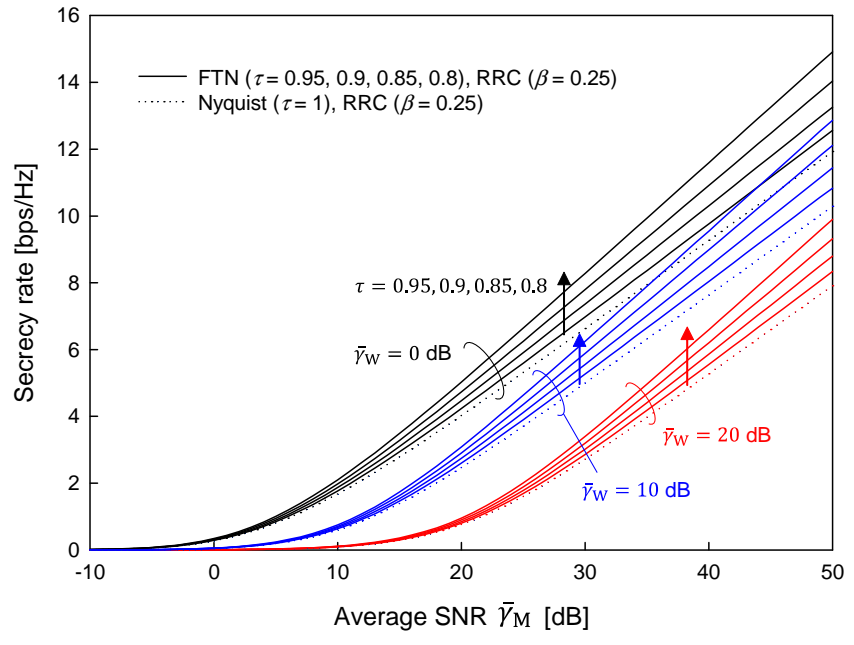

Fig. 5. Numerical average secrecy rate $\mathbb{E}\left[I_{\mathrm{S}}^{\prime}\right] /(B(1+\beta))$ bps/Hz of the proposed E-FTN signaling, employing the RRC shaping filter with the rolloff factor of $\beta=0.25$. The average SNR of the main channel is varied from $\bar{\gamma}_{\mathrm{M}}=-10 \mathrm{~dB}$ to $50 \mathrm{~dB}$. The average SNR of the wiretap channel is set as $\bar{\gamma}_{\mathrm{W}}=0 \mathrm{~dB}, 10 \mathrm{~dB}$, and $20 \mathrm{~dB}$. Packing ratio values from $\tau=0.95$ to 0.8 with the step of 0.05 are plotted, along with the associated mutual information curves of the conventional Nyquist criterion $(\tau=1)$ with the RRC shaping filter. The vertical arrows indicate decreasing $\tau$.

by

$$
I_{\mathrm{S}}^{\prime}=\left\{\begin{array}{cc}
\frac{B}{\tau N}\left[\sum_{i=0}^{N-1} \log _{2}\left(1+\lambda_{i} \gamma_{\mathrm{M}}\right)\right. & \\
\left.-\sum_{i=0}^{N-1} \log _{2}\left(1+\lambda_{i} \gamma_{\mathrm{W}}\right)\right] & \left(\gamma_{\mathrm{M}}>\gamma_{\mathrm{W}}\right) \\
0 & \left(\gamma_{\mathrm{M}} \leq \gamma_{\mathrm{W}}\right)
\end{array}\right.
$$

where power allocation of the proposed E-FTN signaling is deactivated by setting $q_{i}=1$ for $i=1, \cdots, N$. Note that the instantaneous secrecy rate of the conventional FTN benchmark also corresponds to that of the original unprecoded FTN signaling scheme, since EVD-based precoding, as well as weighing, does no change mutual information if power allocation is deactivated [33]. Moreover, unlike in the proposed E-FTN signaling scheme, the secrecy rate in the conventional FTN signaling scheme depends on the block length $N$ and the eigenvalue distribution. The system parameters were the same as those employed in Fig. 3, while the block length was set to $N=5000$. Observe in Fig. 4 that the proposed E-FTN signaling scheme outperformed the conventional FTN benchmark. Upon decreasing the symbol's packing ratio, the performance advantage of the proposed E-FTN signaling scheme increased.

Fig. 5 shows the numerical average secrecy rate $\mathbb{E}\left[I_{\mathrm{S}}^{\prime}\right] /(B(1+\beta))$ bps/Hz of the proposed E-FTN signaling scheme, employing the RRC shaping filter with the roll-off factor of $\beta=0.25$. The average SNR of the main channel was varied from $\bar{\gamma}_{\mathrm{M}}=-10 \mathrm{~dB}$ to $50 \mathrm{~dB}$, while that of the wiretap channel set as $\bar{\gamma}_{\mathrm{W}}=0 \mathrm{~dB}, 10 \mathrm{~dB}$, and $20 \mathrm{~dB}$. The packing ratio was varied from $\tau=0.95$ to 0.8 with the step of 0.05 . The associated numerical curves of the Nyquist-based scheme ( $\tau=1)$, employing the RRC shaping filter, were also plotted. As shown, as the average SNR of the main channel 


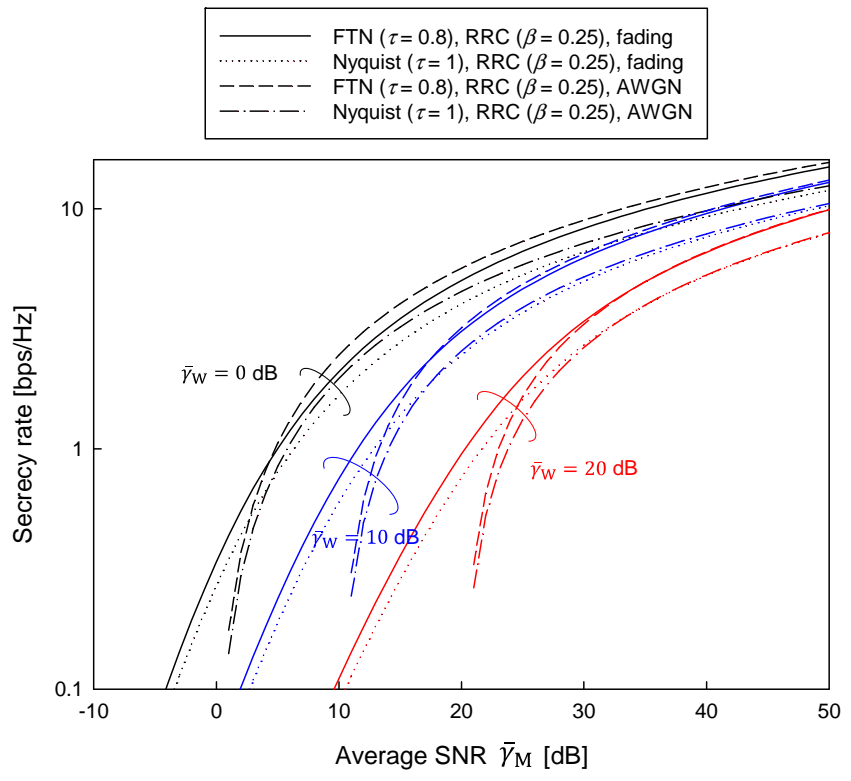

Fig. 6. The average secrecy rate $\mathbb{E}\left[I_{\mathrm{S}}^{\prime}\right] /(B(1+\beta)) \mathrm{bps} / \mathrm{Hz}$ of the proposed E-FTN signaling scheme and that of the Nyquist-based transmission $(\tau=1)$, both employing the RRC shaping filter with the roll-off factor of $\beta=0.25$, considered for the AWGN and Rayleigh fading scenarios. The average SNR of the main channel is varied from $\bar{\gamma}_{\mathrm{M}}=-10 \mathrm{~dB}$ to $50 \mathrm{~dB}$. The wiretap channel is set as $\bar{\gamma}_{\mathrm{W}}=0 \mathrm{~dB}, 10 \mathrm{~dB}$, and $20 \mathrm{~dB}$.

increases, the secrecy rate of E-FTN signaling increases.

Furthermore, Fig. 6 shows the average secrecy rate $\mathbb{E}\left[I_{\mathrm{S}}^{\prime}\right] /(B(1+\beta)) \mathrm{bps} / \mathrm{Hz}$ of the proposed E-FTN signaling scheme and that of the Nyquist-based transmission $(\tau=1)$, both employing the RRC shaping filter with the roll-off factor of $\beta=0.25$, considered for the AWGN and Rayleigh fading scenarios. The average SNR of the main channel was varied from $\bar{\gamma}_{\mathrm{M}}=-10 \mathrm{~dB}$ to $50 \mathrm{~dB}$, while the wiretap channel was set as $\bar{\gamma}_{\mathrm{W}}=0 \mathrm{~dB}, 10 \mathrm{~dB}$, and $20 \mathrm{~dB}$. Here, the theoretical mutual information for the AWGN scenario is simply given by

$$
\begin{aligned}
\bar{I}_{\mathrm{S}} & =I_{\mathrm{S}}^{\prime} \\
& =\max \left(\frac{B}{\tau}\left[\log _{2}\left(1+\bar{\gamma}_{\mathrm{M}}\right)-\log _{2}\left(1+\bar{\gamma}_{\mathrm{W}}\right)\right], 0\right) .
\end{aligned}
$$

Observe in Fig. 6 that the positive average secrecy rate was attained in the entire SNR regime for the fading scenario, owing to the random variations of the channel coefficients. By contrast, the secrecy rate of the proposed scheme remains zero for $\bar{\gamma}_{\mathrm{M}} \leq \bar{\gamma}_{\mathrm{W}}$ in the AWGN scenario, which was the same as in the Nyquist scenario. Hence, fading has the positive effects on the secrecy rate when $\bar{\gamma}_{\mathrm{M}} \leq \bar{\gamma}_{\mathrm{W}}$, as mentioned in [8-10].

Furthermore, Fig. 7 shows the average secrecy rate $\mathbb{E}\left[I_{\mathrm{S}}^{\prime}\right] /(B(1+\beta)) \mathrm{bps} / \mathrm{Hz}$ of the proposed E-FTN signaling scheme and that of the Nyquist-based transmission $(\tau=1)$, both employing the RRC shaping filter, where the roll-off factor was changed from $\beta=0$ to 1 . The average SNR of the main channel and that of the wiretap channel are set to $\bar{\gamma}_{\mathrm{M}}=40 \mathrm{~dB}$ and $\bar{\gamma}_{\mathrm{M}}=0 \mathrm{~dB}$, respectively. The packing ratio of the proposed E-FTN scheme is set to $\tau=1 /(1+\beta)$. Furthermore, the Nyquist pulse transmission with $\beta=0$ corresponds to the unrealistic sinc pulse one. As seen in Fig. 7,

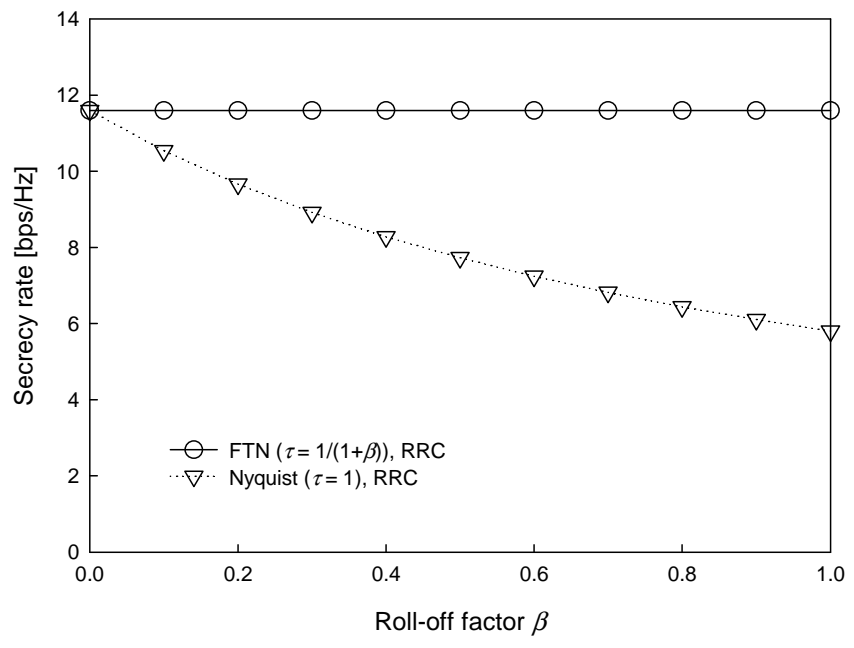

Fig. 7. The average secrecy rate $\mathbb{E}\left[I_{\mathrm{S}}^{\prime}\right] /(B(1+\beta))$ bps/Hz of the proposed E-FTN signaling scheme and that of the Nyquist-based transmission $(\tau=1)$, both employing the RRC shaping filter, where the roll-off factor was changed from $\beta=0$ to 1 . The average SNR of the main channel and that of the wiretap channel are set to $\bar{\gamma}_{\mathrm{M}}=40 \mathrm{~dB}$ and $\bar{\gamma}_{\mathrm{W}}=0 \mathrm{~dB}$, respectively.

the secrecy rate of the proposed E-FTN signaling scheme remains nearly unchanged over the entire $\beta$ regime, while that of the conventional Nyquist pulse transmission decreases upon increasing the roll-off factor. Hence, the higher the roll-off factor, the higher the achievable gain of the proposed scheme over the Nysuist pulse transmission, noting that the roll-off factor $\beta$ is typically determined by the system's requirement.

\section{B. Secrecy Outage Probability}

Fig. 8 shows the theoretical secrecy outage probabilities of the proposed E-FTN signaling with the packing ratio varied from $\tau=0.95$ to 0.8 and the target rate set to $R=10 \mathrm{bps} / \mathrm{Hz}$, calculated according to the derived formula of (71). The average SNR of the main channel was varied from $\bar{\gamma}_{\mathrm{M}}=-20$ $\mathrm{dB}$ to $50 \mathrm{~dB}$, while that of the wiretap channel was maintained to be $\bar{\gamma}_{\mathrm{W}}=0 \mathrm{~dB}$. The associated curves of the conventional Nyquist-based transmission $(\tau=1)$ with the RRC shaping filter and the idealistic rectangular shaping filter (sinc pulse) are also included. The roll-off factor of the RRC filter was given by $\beta=0.25$. Also, the secrecy outage probability, calculated based on Monte Carlo simulations, was also plotted for the validation of each theoretical curve. Observe in Fig. 8 that as the packing ratio $\tau$ decreases, the secrecy outage probability decreases, while outperforming the Nyquist pulse transmission counterpart employing the RRC shaping filter over the entire SNR range. The relative benefit of the proposed E-FTN signaling scheme is especially clear for high SNR values. Note also that the theoretical curves coincided with the numerical curves for each $\tau$ value, hence validating the derived secrecy outage probability formula of (71).

Fig. 9 shows the theoretical secrecy outage probabilities of the proposed E-FTN signaling with the packing ratio varied from $\tau=0.95$ to 0.8 with the step of 0.05 and the average SNRs of the main and wiretap channels set as $\bar{\gamma}_{\mathrm{M}}=40$ 


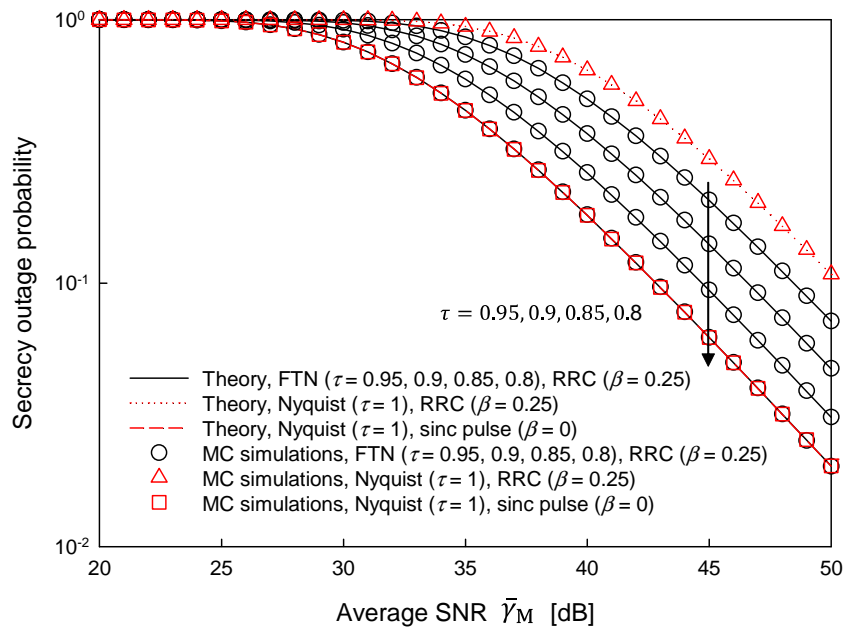

Fig. 8. Theoretical secrecy outage probabilities of the proposed E-FTN signaling, for the packing ratio set to $\tau=0.95,0.9,0.85$, and 0.8 , and the normalized target rate set to $R=10 \mathrm{bps} / \mathrm{Hz}$. The average SNR of the main channel was varied from $\bar{\gamma}_{\mathrm{M}}=-20$ to $50 \mathrm{~dB}$, while that of the wiretap channel is maintained to be $\bar{\gamma}_{\mathrm{W}}=0 \mathrm{~dB}$. The associated curves of the conventional Nyquist-based transmission $(\tau=1)$ with the RRC shaping filter and the idealistic rectangular shaping filter (sinc pulse) are also plotted. The vertical arrow indicates decreasing $\tau$. The associated secrecy outage probabilities calculated based on the Monte Carlo simulations are plotted for validation.

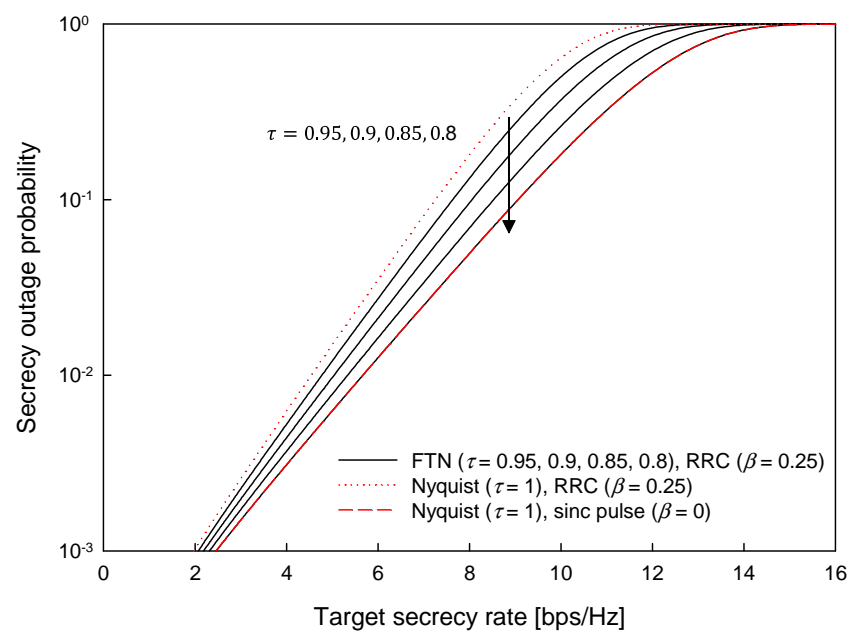

Fig. 9. Theoretical secrecy outage probabilities of the proposed E-FTN signaling for the packing ratio set to $\tau=0.95,0.9,0.85$, and 0.8 and the average SNR of the main and wiretap channels set as $\bar{\gamma}_{\mathrm{M}}=40 \mathrm{~dB}$ and $\bar{\gamma}_{\mathrm{W}}=0 \mathrm{~dB}$, respectively. The target rate was varied from $R=1$ to 16 $\mathrm{bps} / \mathrm{Hz}$. The associated curves of the conventional Nyquist-based transmission $(\tau=1)$ with the RRC shaping filter and the idealistic rectangular shaping filter are also plotted.

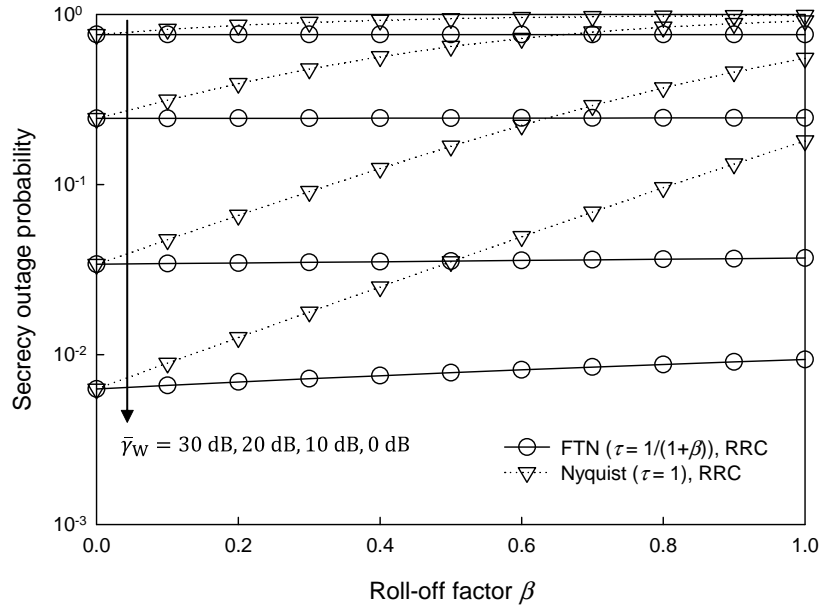

Fig. 10. Theoretical secrecy outage probabilities of the proposed E-FTN signaling scheme and the Nyquist pulse transmission, both employing the RRC shaping filter. The target rate is fixed to $R=5 \mathrm{bps} / \mathrm{Hz}$. The average SNR of the main channel is maintained to be $\bar{\gamma}_{\mathrm{M}}=40 \mathrm{~dB}$, while that of the wiretap channel is given by $\bar{\gamma}_{\mathrm{W}}=30 \mathrm{~dB}, 20 \mathrm{~dB}$, and $10 \mathrm{~dB}$. The packing ratio is set as $\tau=1 /(1+\beta)$. The vertical arrow indicates decreasing $\bar{\gamma}_{\mathrm{W}}$.

$\mathrm{dB}$ and $\bar{\gamma}_{\mathrm{W}}=0 \mathrm{~dB}$, respectively. The roll-off factor of the RRC shaping filter is given by $\beta=0.25$. The target rate was varied from $R=1$ to $16 \mathrm{bps} / \mathrm{Hz}$. The associated curves of the conventional Nyquist-based transmission $(\tau=1)$, employing the RRC shaping filter with the roll-off factor of $\beta=0.25$ and the idealistic rectangular shaping filter $(\beta=0)$, are included. As shown in Fig. 9, it was found that the proposed E-FTN signaling schime exhibited a non-unity outage probability over a wide range of target rates, while outperforming the Nyquist pulse counterpart with the RRC shaping filter. Moreover, as $\tau$ decreased, the achievable secrecy outage probability performance improved. However, as expected from (71), the performance gain of the proposed E-FTN signaling over the conventional Nyquist pulse transmission with the RRC shaping filter in terms of the secrecy outage probability is negligible for $R \ll 1$ or for $\tau R \gg 1$.

Fig. 10 shows the theoretical secrecy outage probabilities of the proposed E-FTN signaling scheme and the Nyquist pulse transmission, both employing the RRC shaping filter. The target rate is fixed to $R=5 \mathrm{bps} / \mathrm{Hz}$. The average SNR of the main channel is maintained to be $\bar{\gamma}_{\mathrm{M}}=40 \mathrm{~dB}$, while that of the wiretap channel is given by $\bar{\gamma}_{\mathrm{W}}=30 \mathrm{~dB}, 20$ $\mathrm{dB}$, and $10 \mathrm{~dB}$. The packing ratio is set as $\tau=1 /(1+\beta)$. Observe in Fig. 10 that while for $\beta=0$, both the proposed E-FTN signaling scheme and the conventional Nyquist pulse transmission exhibited the same performance, the performance advantage of the E-FTN signaling scheme increases upon increasing the roll-off factor $\beta$ in each $\bar{\gamma}_{\mathrm{W}}$ scenario.

Although we focused our attention on the derivation of the achievable secrecy performance bounds of E-FTN signaling in this paper, further investigations are needed for the practical implementation of E-FTN signaling. For example, in our performance results, the symbol packing ratio was limited in the range of $\tau \geq 1 /(1+\beta)$, since this limitation allows us 
to accurately compute all the eigenvalues of the ISI matrix G. Also, the details of the issues, which are related to a high sampling rate, inter-block interference, a high detection complexity, and calculation precision, can be found in [33]. Note that the secrecy performance analysis conducted for EFTN signaling described in this paper is readily applicable to the frequency-domain counterpart [35].

A further secrecy performance improvement may be attainable by reducing mutual information of the wiretap channel, relative to that of the main channel, with the aid of colocated and cooperative beamforming and jamming [3, 41, 42], in addition to the proposed E-FTN signaling scheme. However, detailed investigations of this are beyond the scope of the present study, and are therefore left for the future.

\section{CONCLUSIONS}

In this paper, we investigated the secrecy performance of E-FTN signaling and E-NOFDM in a quasi-static frequencyflat Rayleigh fading channel. The theoretical secrecy mutual information and secrecy outage probability of E-FTN signaling were derived. It was demonstrated that E-FTN signaling and E-NOFDM have the potential of increasing the secrecy performance in comparison to conventional Nyquist-based orthogonal transmission.

\section{REFERENCES}

[1] M. Bloch and J. Barros, Physical-layer security: From information theory to security engineering. Cambridge University Press, 2011.

[2] Y. Shiu, S. Y. Chang, H. Wu, S. C. . Huang, and H. Chen, "Physical layer security in wireless networks: A tutorial," IEEE Wireless Communications, vol. 18, no. 2, pp. 66-74, Apr. 2011.

[3] A. Mukherjee, S. A. A. Fakoorian, J. Huang, and A. L. Swindlehurst, "Principles of physical layer security in multiuser wireless networks: A survey," IEEE Communications Surveys and Tutorials, vol. 16, no. 3, pp. 1550-1573, 2014

[4] Y. Wu, A. Khisti, C. Xiao, G. Caire, K. Wong, and X. Gao, "A survey of physical layer security techniques for 5G wireless networks and challenges ahead," IEEE Journal on Selected Areas in Communications, vol. 36, no. 4, pp. 679-695, Apr. 2018.

[5] J. M. Hamamreh, H. M. Furqan, and H. Arslan, "Classifications and applications of physical layer security techniques for confidentiality: A comprehensive survey," IEEE Communications Surveys and Tutorials, vol. 21 , no. 2 , pp. 1773-1828, 2019.

[6] A. D. Wyner, "The wire-tap channel," Bell System Technical Journal, vol. 54, no. 8, pp. 1355-1387, 1975.

[7] S. Leung-Yan-Cheong and M. Hellman, "The Gaussian wire-tap channel," IEEE Transactions on Information Theory, vol. 24, no. 4, pp. 451456, July 1978.

[8] Y. Liang, H. V. Poor, and S. Shamai, "Secure communication over fading channels," IEEE Transactions on Information Theory, vol. 54, no. 6, pp. 2470-2492, May 2008.

[9] P. K. Gopala, L. Lai, and H. El Gamal, "On the secrecy capacity of fading channels," IEEE Transactions on Information Theory, vol. 54 no. 10 , pp. 4687-4698, Sept. 2008.

[10] M. Bloch, J. Barros, M. R. D. Rodrigues, and S. W. McLaughlin, "Wireless information-theoretic security," IEEE Transactions on Information Theory, vol. 54, no. 6, pp. 2515-2534, June 2008.

[11] A. O. Hero, "Secure space-time communication," IEEE Transactions on Information Theory, vol. 49, no. 12, pp. 3235-3249, Dec. 2003.

[12] T. Liu and S. Shamai, "A note on the secrecy capacity of the multipleantenna wiretap channel," IEEE Transactions on Information Theory, vol. 55, no. 6, pp. 2547-2553, June 2009.

[13] A. Khisti and G. W. Wornell, "Secure transmission with multiple antennas - part II: The MIMOME wiretap channel," IEEE Transactions on Information Theory, vol. 56, no. 11, pp. 5515-5532, Nov. 2010.

[14] F. Oggier and B. Hassibi, "The secrecy capacity of the MIMO wiretap channel," IEEE Transactions on Information Theory, vol. 57, no. 8, pp. 4961-4972, Aug. 2011.
[15] - "A perspective on the MIMO wiretap channel," Proceedings of the IEEE, vol. 103, no. 10, pp. 1874-1882, Oct. 2015.

[16] X. Chen, D. W. K. Ng, W. H. Gerstacker, and H. Chen, "A survey on multiple-antenna techniques for physical layer security," IEEE Communications Surveys and Tutorials, vol. 19, no. 2, pp. 1027-1053, 2017.

[17] J. B. Anderson, F. Rusek, and V. Owall, "Faster-than-Nyquist signaling," Proceedings of the IEEE, vol. 101, no. 8, pp. 1817-1830, Aug. 2013.

[18] J. Fan, S. Guo, X. Zhou, Y. Ren, G. Y. Li, and X. Chen, "Faster-thanNyquist signaling: An overview," IEEE Access, vol. 5, pp. 1925-1940, 2017.

[19] T. Ishihara and S. Sugiura, "Iterative frequency-domain joint channel estimation and data detection of faster-than-Nyquist signaling," IEEE Transactions on Wireless Communications, vol. 16, no. 9, pp. 62216231, Sept. 2017

[20] T. Ishihara and S. Sugiura, "Faster-than-Nyquist signaling with index modulation," IEEE Wireless Communications Letters, vol. 6, no. 5, pp. 630-633, Oct. 2017.

[21] W. Yuan, N. Wu, A. Zhang, X. Huang, Y. Li, and L. Hanzo, "Iterative receiver design for FTN signaling aided sparse code multiple access," IEEE Transactions on Wireless Communications, vol. 19, no. 2, pp. 915928, Feb. 2020

[22] F. Rusek and J. B. Anderson, "Non binary and precoded faster than Nyquist signaling," IEEE Transactions on Communications, vol. 56, no. 5, pp. 808-817, May 2008.

[23] Y. G. Yoo and J. H. Cho, "Asymptotic optimality of binary faster-thanNyquist signaling," IEEE Communications Letters, vol. 14, no. 9, pp. 788-790, Sept. 2010.

[24] A. Gattami, E. Ringh, and J. Karlsson, "Time localization and capacity of faster-than-Nyquist signaling," in IEEE Global Communications Conference, San Diego, CA, USA, Dec. 2015, pp. 1-7.

[25] I. Darwazeh, T. Xu, T. Gui, Y. Bao, and Z. Li, "Optical SEFDM system; bandwidth saving using non-orthogonal sub-carriers," IEEE Photonics Technology Letters, vol. 26, no. 4, pp. 352-355, Feb. 2014.

[26] S. V. Zavjalov, S. V. Volvenko, and S. B. Makarov, "A method for increasing the spectral and energy efficiency SEFDM signals," IEEE Communications Letters, vol. 20, no. 12, pp. 2382-2385, Dec. 2016.

[27] T. Xu and I. Darwazeh, "Transmission experiment of bandwidth compressed carrier aggregation in a realistic fading channel," IEEE Transactions on Vehicular Technology, vol. 66, no. 5, pp. 4087-4097, May 2017.

[28] M. Nakao and S. Sugiura, "Spectrally efficient frequency division multiplexing with index-modulated non-orthogonal subcarriers," IEEE Wireless Communications Letters, vol. 8, no. 1, pp. 233-236, Feb. 2019.

[29] S. Osaki, M. Nakao, T. Ishihara, and S. Sugiura, "Differentially modulated spectrally efficient frequency-division multiplexing," IEEE Signal Processing Letters, vol. 26, no. 7, pp. 1046-1050, July 2019.

[30] Y. Ma, N. Wu, W. Yuan, D. W. K. Ng, and L. Hanzo, "Joint channel estimation and equalization for index-modulated spectrally efficient frequency division multiplexing systems," IEEE Transactions on Communications, 2020, in press.

[31] J. Wang, W. Tang, X. Li, and S. Li, "Filter hopping based faster-thanNyquist signaling for physical layer security," IEEE Wireless Communications Letters, vol. 7, no. 6, pp. 894-897, Dec. 2018.

[32] Y. Li, J. Wang, W. Tang, X. Li, and S. Li, "A variable symbol duration based FTN signaling scheme for PLS," in International Conference on Wireless Communications and Signal Processing, Xi'an, China, Oct. 2019, pp. $1-5$.

[33] T. Ishihara and S. Sugiura, "SVD-precoded faster-than-Nyquist signaling with optimal and truncated power allocation," IEEE Transactions on Wireless Communications, vol. 18, no. 12, pp. 5909-5923, Dec. 2019.

[34] S. Osaki, T. Ishihara, and S. Sugiura, "Non-orthogonal frequencydivision multiplexing based on eigenvalue decomposition," in IEEE Vehicular Technology Conference (VTC2020-Fall), Nov. 2020, pp. 15.

[35] $\longrightarrow$, "Eigenvalue-decomposition-precoded ultra-dense non-orthogonal frequency-division multiplexing," IEEE Transactions on Wireless Communications, vol. 19, no. 12, pp. 8165-8178, Dec. 2020.

[36] Y. J. D. Kim, "Properties of faster-than-Nyquist channel matrices and folded-spectrum, and their applications," in IEEE Wireless Communications and Networking Conference, Doha, Qatar, Apr. 2016, pp. 1-7.

[37] B. Farhang-Boroujeny, "OFDM versus filter bank multicarrier," IEEE Signal Processing Magazine, vol. 28, no. 3, pp. 92-112, May 2011.

[38] D. Rainnie, Y. Feng, and J. Bajcsy, "On capacity merits of spectrally efficient FDM," in IEEE Military Communications Conference, Tampa, FL, USA, Oct. 2015, pp. 581-586.

[39] A. Goldsmith, Wireless communications. Cambridge University Press, 2005. 
[40] K. Masaki, T. Ishihara, and S. Sugiura, "Tradeoff between calculation precision and information rate in eigendecomposition-based faster-thanNyquist signaling," IEEE Access, vol. 8, pp. 223 461-223 471, 2020.

[41] L. Dong, Z. Han, A. P. Petropulu, and H. V. Poor, "Improving wireless physical layer security via cooperating relays," IEEE Transactions on Signal Processing, vol. 58, no. 3, pp. 1875-1888, Mar. 2010.

[42] R. Nakai and S. Sugiura, "Physical layer security in buffer-state-based max-ratio relay selection exploiting broadcasting with cooperative beamforming and jamming," IEEE Transactions on Information Forensics and Security, vol. 14, no. 2, pp. 431-444, Feb. 2019. 